\title{
Neolignans from the Arils of Myristica fragrans as Potent Antagonists of CC Chemokine Receptor 3
}

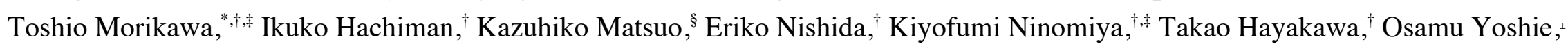
Osamu Muraoka, ${ }^{\dagger, *}$ and Takashi Nakayama, ${ }^{*}$

${ }_{\dagger}^{\dagger}$ Pharmaceutical Research and Technology Institute, ${ }^{\star}$ Antiaging Center, and ${ }^{\S}$ Faculty of Pharmacy, Kindai University, 3-4-1 Kowakae, Higashi-osaka, Osaka 577-8502, Japan

${ }^{\star}$ Faculty of Medicine, Kindai University, 377-2 Ohno-higashi, Osaka-sayama, Osaka 589-8511, Japan

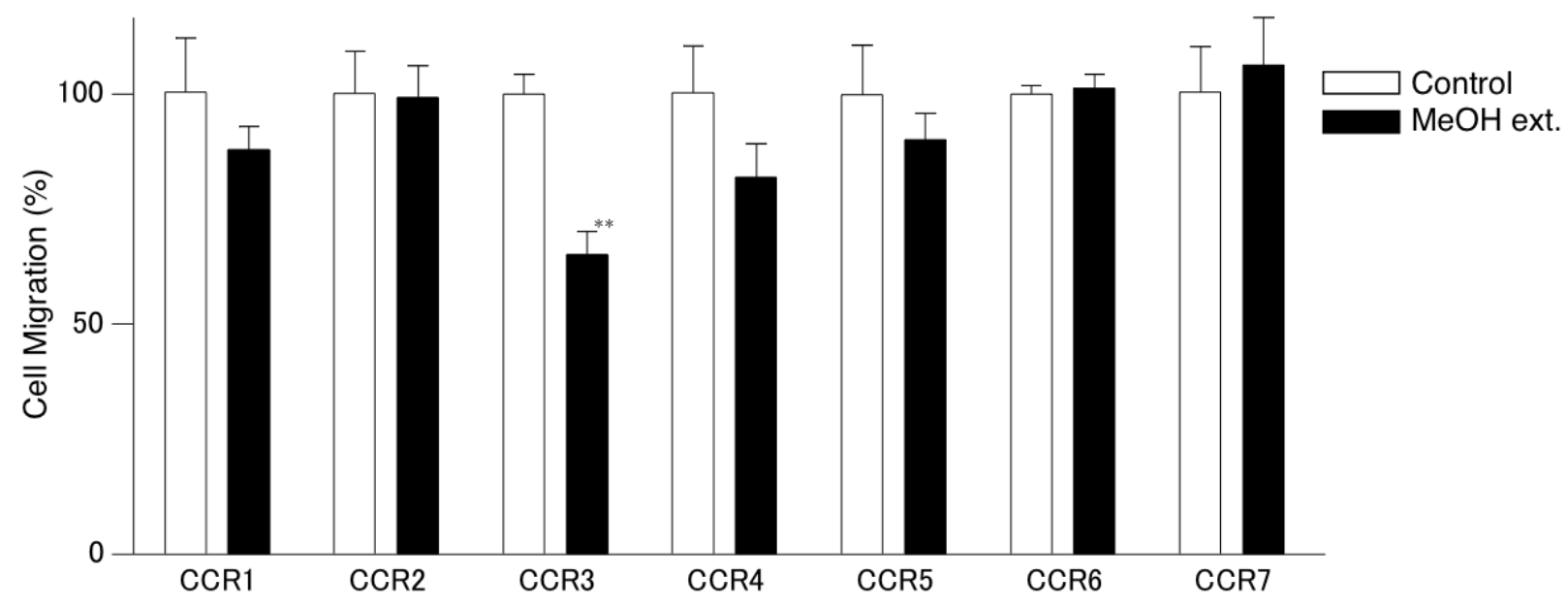

Figure S1. Effects of the methanol extract of M. fragrans arils on chemokine-induced chemotaxis in L1.2 cells expressing CCR1-7. Each experiment was performed using a concentration of $100 \mu \mathrm{g} / \mathrm{mL}$. Migration of L1.2-CCR1-7 cells was induced by $10 \mathrm{nM}$ CCL3, CCL2, CCL11, CCL22, CCL3, CCL20, and CCL21. Results from three experiments are shown as mean \pm SEM. Significant difference was analyzed by Student's $t$ test, ${ }^{* *} p<0.01$ (vs. control). 

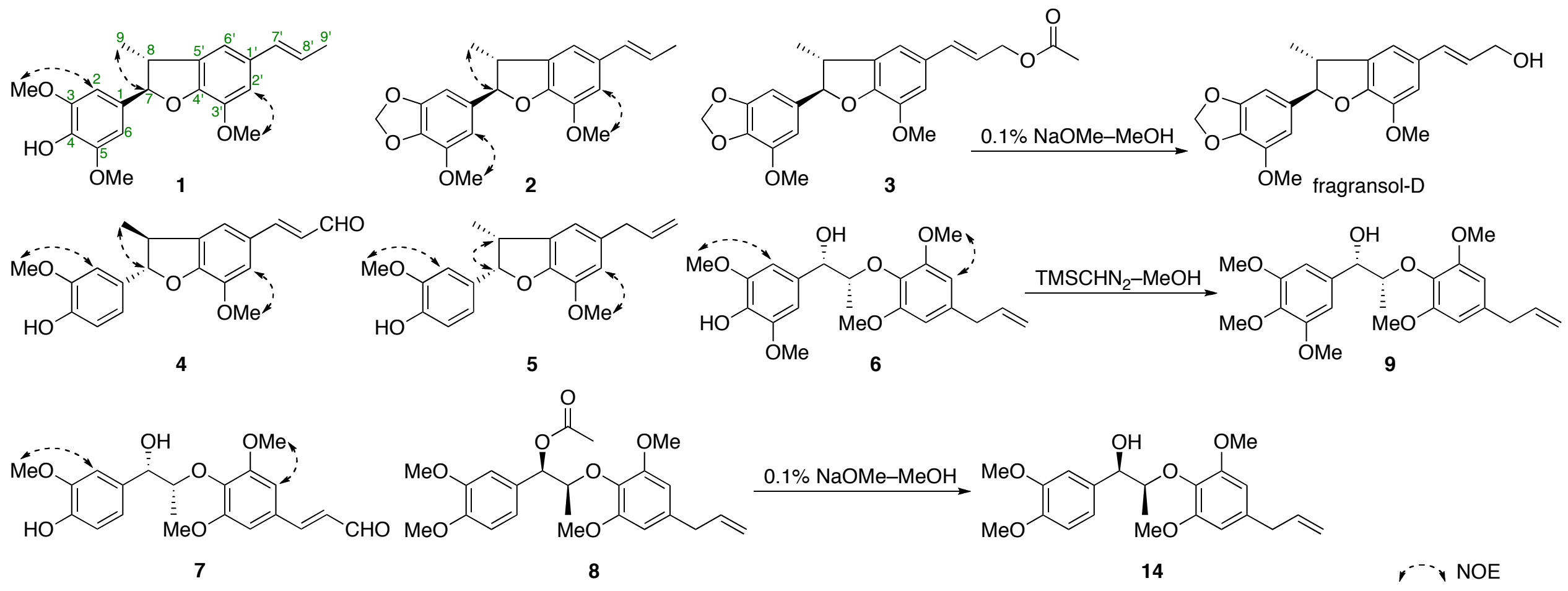

Figure S2. Stereostructures of 1-8 